J. Lake Sci. (湖泊科学), $2007, \mathbf{1 9}(4): 445-450$

http://www. jlakes.org. E-mail: jlakes@ niglas. ac.cn

(c) 2007 by Journal of Lake Sciences

\title{
混合禁忌搜索算法在湖泊富营养化评价中的应用
}

\author{
汪嘉杨, 李祚泳, 熊建秋, 徐婷婷
}

(成都信息工程学院,成都 610041)

摘 要: 本文提出了幂函数加和型湖泊富营养化评价指数公式,同时将禁忌搜索算法与模拟退火、免疫进化算法有机地结 合起来,建立了求解优化问题的混合禁忌搜索算法 (HTS). 将此算法用于评价指数公式的参数优化,从而建立起湖泊富 营养化综合评价模型, 为湖泊富营养化评价和预测提供了一条有效的途径. 将优化好的湖泊富营养化模型用于东湖富营 养化评价的实例研究,结果表明此评价模型能较好地反映湖泊富营养化水平,并具有较好的通用性和实用性.

关键词 : 湖泊;富营养化;评价;禁忌搜索;模拟退火;免疫算法

\section{Application of Hybrid Taboo Search to lake eutrophication evaluation}

WANG Jiayang, LI Zuoyong, XIONG Jianqiu \& XU Tingting

(Chengdu University of Information Technology, Chengdu 610041,P. R. China)

\begin{abstract}
The article presents a comprehensive index formula with power function for evaluating lake eutrophication, and the Hybrid Taboo Search (HTS) algorithm was also presented in order to optimize the parameters in the formula. Here, Hybrid Taboo Search is set up through combining Taboo Search with Simulated Anneal and Immune Arithmetic. The optimized formula was used in evaluating the level of ten sections in Donghu Lake. The results show that the model can describe the nature level of eutrophication well, and has indicatmg availability and simplicity for evaluation.
\end{abstract}

Keywords: Lake; eutrophication; evaluation; Taboo Search; Simulated Annealing; Immune Arithmetic

近百年来, 由于经济发展, 人口膨胀, 以及对自然资源不合理的开发利用,全球水体富营养化问题日益 严重, 尤其是湖泊生态系统结构遭到空前的破坏、生态功能萎缩, 造成了巨大的经济损失. 湖泊的富营养化 已成为国际上普遍存在的问题，也是国际湖泊学界关注的焦点问题之一. 因此,为了确定湖泊富营养化的 程度, 准确反映水环境的质量和污染状况, 并预测将来的发展趋势, 对湖泊水体的营养状况进行综合评价和 预测成为十分重要和关键的一项内容. 湖泊富营养化评价的目的是为开展环境污染综合治理、环境规划及 管理提供科学依据 ${ }^{[1]}$.

从 20 世纪 70 年代初期开展富营养化研究至今,湖泊富营养化模型取得了飞速的发展. 国内外先后提 出了特征法、参数法、营养状态指数法、生物评价法和评分法等多种评价方法,近年来发展起来的模糊集理 论、灰色系统、遗传算法和人工神经网络等方法也已用于水体富营养化的评价与预测 ${ }^{[2-7]}$. 然而, 由于湖泊 是一个复杂、动态的生态系统, 水体富营养化的变化特性和规律错综复杂, 其评价方法和指标体系仍处于不 断探索和发展之中,至今尚未形成一种公认的理论体系和方法体系,要建立一种简单而又能反映湖泊富营 养化发展普遍规律的评价模型难度较大. 为此, 本文提出形式简单的幂函数加和型湖泊富营养化评价指数 公式,并采用混合禁忌搜索算法 (Hybrid Taboo Search, HTS) 对其中的参数进行优化,从而建立起湖泊富营 养化综合评价模型, 为水体富营养化评价和预测提供了一条有效的途径.

禁忌搜索算法 (Taboo Search, TS) 是一种有目的的启发式搜索法,它与局部优化法相比陷人局部极小

* 国家“973”重大基础研究项目 (2002CB412301),成都信息工程学院科技发展基金(CSRF200501) 联合资助. 2006 05-29 收稿; 2006-10-17 收修改稿. 汪嘉杨,女,1980 年生, 硕士研究生; E-mail : lizuoyong@ cuit. edu. cn. 
值的机率很小, 比遗传算法、模拟退火法更易利用问题的特殊信息,因此它具有更强的全局搜索能力, 在复 杂和大型问题上有独特的效果, 已成功地应用于资源规划、超大规模集成电路设计、通讯等领域,并广泛地 应用于旅行商 (TSP) 、车间调度问题及目标函数的优化问题 ${ }^{[8-12]}$. 但对于连续优化问题来说即使解空间很 小,其可能的解的数目都是无限多的,按照求解组合优化问题的优化方法不加以改进来求解连续优化问题 达不到理想的效果. 因此,对基本的禁忌搜索算法的改进是十分必要的. 本文将禁忌搜索算法与模拟退火 (Simulated Annealing, SA)、免疫进化算法 (Immune Evolotionary Arithmetic, IEA) 有机地结合起来,建立了求 解优化问题的混合禁忌搜索算法,算法精度高,性能稳定,搜索迅速.

\section{1 用于湖泊富营养化评价公式参数优化的混合禁忌搜索算法}

\section{1 禁忌搜索算法的基本原理}

$\mathrm{TS}$ 的基本思想是 ${ }^{[12]}$ : 假设给出一个解邻域,首先在解邻域中找出一个初始局部解 $x$ 作为当前解, 并令 当前解为最优解, 然后以这个当前解 $x$ 作为起点, 在解邻域中搜索最优解 $x$ '. 当然, 这个最优解可能与前一 次所得的最优解相同, 为了避免这种循环现象的出现, 设置一个记忆近期操作的禁忌表,如果当前的搜索操 作是记录在此表中的操作,那么这一搜索操作就被禁止; 否则以 $x$ '取代 $x$ 作为当前解. 但禁忌表有可能限 制某些可以导致更好解的“移动”, 此时 TS 算法可以用期望准则进行解禁, 以便迅速找到更好的解.

\section{2 模拟退火算法的基本原理}

模拟退火算法是基于蒙特卡罗迭代求解法的一种启发式随机搜索算法. 算法通过设定一初温和初态, 伴随温度的不断下降,结合概率突跳特性在解空间中通过邻域函数进行随机搜索,最终得到全局最优.

\section{3 混合禁忌搜索算法}

HTS 有两点改进:一是采用蒙特卡罗准则作为禁忌搜索算法中接受非禁忌劣解的概率, 从而使禁忌搜 索算法有指导地跳出局部最优; 二是采用免疫进化算法的进化特征生成禁忌搜索迭代继续的邻域空间.

1.3.1 禁忌搜索与模拟退火算法的结合 TS 算法通过禁忌列表的使用,尽量避免迂回搜索, 允许接受邻域 中非禁忌的劣解和满足藐视准则的优良解, 是 TS 算法区别于传统局部邻域搜索算法的关键, 也是影响搜索 速度重要因素. 另一方面, 由于模拟退火使用蒙特卡罗准则, 能以一定的概率接受目标函数值不太好的状 态, 理论上经过足够长的时间后也可跳出来, 从而收玫到全局最优解. 但是, 由于求解时间长同时也很难搜 索到满意的优秀解. 因此, HTS 将两种算法相结合, 采用 SA 中的蒙特卡罗准则指导 TS 算法中接受非禁忌 劣解的概率选择, 使 HTS 提高传统 TS 算法的 “下山” 能力的同时, 提高 TS 算法的搜索速度和精度. 因此, 寻优过程中非禁忌劣解, 按照蒙特卡罗准则作为此非禁忌劣解的接受概率 $P$ :

$$
P_{i}(i \Rightarrow j)=\left\{\begin{array}{cl}
1 & f(j)=f(i) \\
\exp \left[\frac{-\Delta f}{t}\right] & f(j)>f(i)
\end{array}\right.
$$

确定是否接受从当前解 $i$ 到新解 $j$ 的转移.

1.3 .2 禁忌搜索算法中邻域解的生成 邻域集为可应用于当前解的移动的集合. 较好的邻域的确定能大大 提高搜索的速度,避免局部重复搜索, 是影响禁忌搜索算法质量和效率的关键因素. 通常是随机生成邻域 移动, 或者设定特定的步长以进行移动. 前种方法具有很大的随机性, 容易使搜索算法寻优过程增加; 后种 方法具有很强的主观因素, 如果步长设置值较大, 容易跳过优秀解, 如果步长设置值较小, 容易陷人局部寻 优. HTS 采用免疫思想指导的邻域结构,使得通过几步迭代就可以对较大的邻域进行搜索, 提高搜索速度. 其具体做法为: 在选定的搜索子空间内,利用免疫进化算法的进化特征生成禁忌搜索迭代继续的邻域空间. 免疫进化算法描述为 ${ }^{[13]}$ :

$$
\left\{\begin{array}{l}
a_{i}^{t+1}=a_{i, b e s t}^{t}+\sigma_{i}^{t} \times N(0,1) \\
\sigma_{i}^{t+1}=\sigma_{\varepsilon}+\sigma_{i}^{0} \times \mathrm{e}^{-A \times t / T}
\end{array} \quad(i=1,2, \cdots)\right.
$$

式中, $a_{i}^{t+1}$ 为子代个体第 $i$ 个分量; $a_{i, \text { best }}^{t}$ 为父代最优个体第 $i$ 个分量 $; \sigma_{i}^{t+1}$ 为子代群体第 $i$ 个分量的标准差; $\sigma_{i}^{t}$ 为父代群体第 $i$ 个分量的标准差; $A$ 为标准差动态调整系数,通常 $A \in[1,10] ; \sigma_{\varepsilon}$ 为标准差基数,通常取 $\sigma_{\varepsilon}=0 ; \sigma_{i}^{0}$ 为对应于初始群体第 $i$ 个分量的标准差; $T$ 为总的进化代数; $t$ 为进化的代数; $N(0,1)$ 为服从标准 
正态分布的随机数.

\section{2 混合禁忌搜索算法流程图}

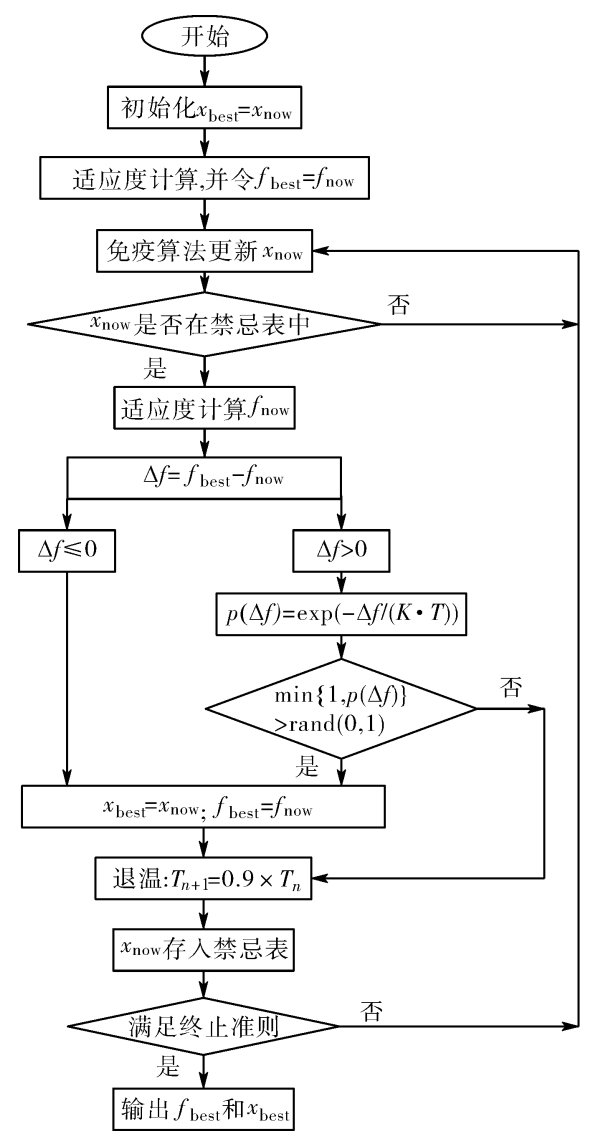

图 1 混合禁忌搜索算法流程图

Fig. 1 Flow chart of HTS

\section{3 基于混合禁忌搜索算法的湖泊富营养化综合评价模型}

\section{1 湖泊富营养化评价指数公式}

湖泊富营养化评价是一个具有不确定性、复杂的系统, 可用幂函数加和型指数公式进行评价：

$$
P I=\alpha \times\left[\sum_{i}^{n} W_{i} \times I_{i}\right]^{\beta}
$$

式中, $P I$ 为湖泊富营养化水平的评价指数; $\alpha, \beta$ 为待优化的参数; $W_{i}$ 为加权数, 满足 $\sum_{i=1}^{n} W_{i}=1$, 通常情况下, 为了简化, 公式中各指标的权值都视为等权的, 因此, $\sum_{i=1}^{n} W_{i} I_{i}=\frac{\sum_{i=1}^{n} I_{i}}{n}$, 即所有指标的指数平均值即可; $n$ 为指 标数目; $I_{i}$ 为指标 $i$ 的指数,表示湖泊富营养化指标的发展程度, 由下式确定:

$$
I_{i}= \begin{cases}c_{i} / c_{\max } & \text { 当 } i \text { 为正向指标时 } \\ c_{\text {max }} / c_{i} & \text { 当 } i \text { 为逆向指标时 }\end{cases}
$$

式中, $c_{i}$ 为指标 $i$ 的实际值 (或现状值); $c_{\text {max }}$ 为第 $i$ 个指标的样本原始最大值; $c_{\text {min }}$ 为第 $i$ 个指标的样本原始最 
小值.

\section{2 基于 HTS 优化确定参数 $\alpha$ 和 $\beta$}

式 (3) 中的参数 $\alpha 、 \beta$ 需要根据具体的评价指标体系加以确定,并采用一定的优化算法得到最后的普适 性的评价公式. 本文采用 HTS 优化式 (3) 中的参数 $\alpha$ 和 $\beta$, 并构造目标函数为:

$$
\min f(\alpha, \beta)=\frac{1}{m \times K} \sum_{m, k}\left(P I_{K}-P I_{k 0}\right)^{2}
$$

式中, $m$ 为每个等级样本数目; $K$ 为分级数, (本文 $K=5$ ); $P I_{k}$ 为对于划定的等级 $k$ 的湖泊富营养化水平评价 值; $P I_{k 0}$ 为目标指数值, 根据具体的问题和需要所规定的评价等级目标取值. 按 “等比赋值,等差分级” 原则 划分为 $k=1-5$ 共 5 个级别, 其中任意相邻两级 $P I_{k 0}$ 之比为 $\delta=(0.9 / 0.1)^{1 / 5} \approx 1.552$, 其 5 个评价等级的目 标值 $P I_{k 0}$ 如表 1 所示.

\section{3 湖泊富营养综合评价公式评价指标和评价标准的选择}

湖泊富营养化现象的发生主要是由于水体中的氮、磷营养元素增多的原因所致,造成水体水生生物异 常, 因此在湖泊富营养化的描述中常选择 Chl. a、TP、COD $\mathrm{Mn}_{\mathrm{n}} 、 \mathrm{BOD}_{5} 、 \mathrm{NH}_{3}-\mathrm{N}$ 五个项目作为评价指标. 目前国 内尚无统一的富营养化评价标准, 根据日本相畸守弘等人修正的营养状态指数 ${ }^{[14]}$, 参照国内部分湖泊、水 库营养状态评价标准 ${ }^{[15,16]}$, 并参照国家地面水环境质量标准 (GB3838-88), 制定湖泊水体富营养化评价 标准 (表 1 ).

表 1 湖泊富营养化评价评分标准及优化后各个评价等级结果分析

Tab. 1 Grading standards of eutrophication index evaluation and analyses of optimized appraise results

\begin{tabular}{cccccc}
\hline 评价等级 & 极贫营养化 $(\mathrm{I})$ 贫营养化 ( II ) & 中营养化 $(\mathrm{III})$ & 富营养化 $(\mathrm{IV})$ & 重富营养化 $(\mathrm{V})$ \\
\hline $\mathrm{Chl} . \mathrm{a}(\mu \mathrm{g} / \mathrm{L})$ & 0.2500 & 1.5900 & 10.0000 & 63.1000 & 398.0000 \\
$\mathrm{TP}(\mathrm{mg} / \mathrm{L})$ & 0.0050 & 0.0190 & 0.0650 & 0.2230 & 0.7600 \\
$\mathrm{COD}_{\mathrm{Mn}}(\mathrm{mg} / \mathrm{L})$ & 1.4000 & 2.9600 & 6.2900 & 13.3300 & 28.2500 \\
$\mathrm{BOD}_{5}(\mathrm{mg} / \mathrm{L})$ & 0.6500 & 1.4100 & 3.0600 & 6.6400 & 14.4200 \\
$\mathrm{NH}_{3}-\mathrm{N}(\mathrm{mg} / \mathrm{L})$ & 0.0170 & 0.0550 & 0.1860 & 0.6300 & 2.1100 \\
$P I_{k 0}$ & 0.1552 & 0.2408 & 0.3737 & 0.5799 & 0.9000 \\
$P I_{k}$ & 0.1535 & 0.2326 & 0.3583 & 0.5643 & 0.9109 \\
$\varepsilon$ & 0.0017 & 0.0082 & 0.0154 & 0.0156 & 0.0109 \\
$\varepsilon$ & 0.0110 & 0.0341 & 0.0412 & 0.0269 & 0.0121 \\
\hline
\end{tabular}

在各指标的各评价等级标准的一定范围内随机选取 10 组数据,作为湖泊富营养化评价的 10 个样本数 据, 共有 50 个样本数据. 将 50 个样本经过式 (4) 归范化处理后的评价指标数据代人目标函数式 (5) 中, 在 满足式 (5) 的目标准则条件下, 用 HTS 算法对式 (3) 中的系数 $\alpha 、 \beta$ 反复寻优, 并在微机上用 Matlab 编程实 现,设定优化计算采用的主要参数如表 2 示. 当优化目标函数值 $f(\alpha 、 \beta) \leqslant 0.05$ 时,输出参数 $\alpha=1.8562, \beta$ $=0.4251$, 得到湖泊富营养化评价公式为:

$$
P I=1.8562 \times\left[\sum_{i=1}^{n} W_{i} \times P I_{i}\right]^{0.4251}
$$

表 2 混合禁忌搜索算法参数设置

Tab. 2 Set parameters of HTS

\begin{tabular}{cccccc}
\hline 参数 & 禁忌列表长度 & 初始温度 $\left(T_{0}\right)$ & 终止温度 $(T)$ & 退火系数 $(r)$ & 同温度下循环次数 \\
\hline 数值 & 10 & 100000 & 1 & 0.9 & 1000 \\
\hline
\end{tabular}


营养化的评价分级标准, 并对每个等级优化结果进行误差分析, 分别计算绝对误差 $\varepsilon$ 和相对误差 $\varepsilon^{\prime}$, 亦如表 1 所示.

结果分析:采用改进的禁忌搜索算法对目标函数进行优化,50个样本按照式 (6) 计算的误差仅为 0.05 , 各个等级的绝对误差和相对误差均小于 0.05 , 表明此模型总体拟合较好. 进行湖泊富营养化等级评价的时 候, 是采用优化后的评价值作为划分评价等级的依据, 并不采用目标值作为划分评价等级的依据, 因此, 实 际评价的时候, 不会存在优化模型时的误差. 采用优化后的评价公式进行湖泊富营养化评价, 能反映出待 评价湖泊真实水平.

\section{4 实例研究——东湖湖泊富营养状况评价}

进行东湖富营养化状况评价实例研究,评价数据采用东湖 2001 年 4 月至 2002 年 2 月枯、平、丰三个水 期的监测结果, 其中十个湖区的五项评价指标监测值的平均值见表 $3^{[15]}$. 将各个湖泊的实测指标数据经过 式 (4) 处理后输人评价公式 (6) 中, 得到 10 个湖区富营养化评价值, 如表 3 所示, 并进行结果比较与分析. 表 3 同时列出了用营养状态指数法 ${ }^{[15]}$ 和本文方法对 10 个湖区富营养化程度的评价结果.

表 3 东湖十湖区水质评价监测数据

Tab. 3 Water quality-monitoring data of ten sections in Donghu Lake

\begin{tabular}{|c|c|c|c|c|c|c|c|c|}
\hline 湖区名 & $\begin{array}{c}\text { Chl. a } \\
(\mu \mathrm{g} / \mathrm{L})\end{array}$ & $\begin{array}{c}\mathrm{TP} \\
(\mathrm{mg} / \mathrm{L})\end{array}$ & $\begin{array}{c}\mathrm{COD}_{\mathrm{Mn}} \\
(\mathrm{mg} / \mathrm{L})\end{array}$ & $\begin{array}{c}\mathrm{BOD}_{5} \\
(\mathrm{mg} / \mathrm{L})\end{array}$ & $\begin{array}{l}\mathrm{NH}_{3}-\mathrm{N} \\
(\mathrm{mg} / \mathrm{L})\end{array}$ & $\frac{\text { 基于 } \mathrm{HT} \mathrm{S}}{\text { 评价值 }}$ & $\frac{\text { 的评价指数法 }}{\text { 评价等级 }}$ & $\begin{array}{l}\text { 营养状态 } \\
\text { 指数法 }{ }^{*}\end{array}$ \\
\hline 郭郑湖 - 水 & 50.23 & 0.22 & 7.94 & 8.47 & 1.84 & 0.6053 & 重富营养化 & 富营养化 \\
\hline 郭郑湖 - 磨 & 28.16 & 0.08 & 5.67 & 6.03 & 0.50 & 0.4467 & 富营养化 & 富营养化 \\
\hline 汤菱湖 & 8.19 & 0.049 & 3.49 & 2.74 & 0.31 & 0.3374 & 中营养化 & 中营养化 \\
\hline 庙湖 & 42.47 & 0.52 & 9.75 & 12.49 & 5.63 & 0.7839 & 重富营养化 & 富营养化 \\
\hline 后湖 & 12.07 & 0.05 & 5.15 & 2.36 & 0.67 & 0.3831 & 富营养化 & 中营养化 \\
\hline 筲箕湖 & 18.57 & 0.13 & 5.43 & 4.98 & 0.49 & 0.4393 & 富营养化 & 富营养化 \\
\hline 小潭湖 & 5.26 & 0.068 & 5.35 & 2.20 & 0.34 & 0.3494 & 中营养化 & 中营养化 \\
\hline 牛巢湖 & 12.46 & 0.06 & 5.37 & 2.84 & 0.33 & 0.3682 & 富营养化 & 富营养化 \\
\hline 水果湖 & 83.86 & 0.54 & 11.08 & 12.55 & 6.29 & 0.8306 & 重富营养化 & 富营养化 \\
\hline 喻家湖 & 59.61 & 0.17 & 11.30 & 8.42 & 3.30 & 0.6654 & 重富营养化 & 富营养化 \\
\hline
\end{tabular}

* 文献 $[15]$ 中湖泊富营养化状态为贫、中、富 3 个等级.

结果与讨论: (1) 由于文献 [15]采用营养状态指数法, 只对湖泊富营养化等级划分为贫、中、富 3 个等 级, 本文划分为极贫、贫、中、富、重富 5 个等级, 将文献中的贫营养等级划分为极贫和贫营养两级, 富营养化 等级划分为重富和富营养两级,更细淢、合理地表明了东湖各个湖区的富营养化状态.

(2) 表 3 中, 水果湖各项评价指标原始值在待评价的 10 个湖区里最大, 并远远大于前面 8 个湖泊, 因此, 本文将其划分为重富营养化等级更能突出水果湖区的水质状态, 突出东湖富营养化治理的重点. 水果湖、 郭郑湖一水、喻家湖、庙湖四个湖区为重富营养化等级, 是在文献 [15]的富营养化等级基础上的细致划分, 其评价结果是一致的.

(3) 从评价结果我们可以看出, 水果湖、郭郑湖一水、喻家湖、庙湖四个湖区为重富营养化等级, 郭郑 湖一磨、筲箕湖、后湖、牛巢湖四个湖区为富营养化等级, 小潭湖、汤菱湖两个湖区为中营养化等级, 而贫营 养的水体则不存在. 富营养化严重的水体位于人口稠密区、养殖区和湖汉处,并且其分布在平面上很不均 匀,其程度自西向东递减.

(4) 通过表 3 比较和实际情况分析表明,用本文方法得到的评价结果能客观反映湖泊水体的富营养化 水平, 并能更好地区分各个营养状态, 突出富营养化治理的重点区域,为水环境管理决策提供科学依据. 


\section{5 结论}

本文采用混合禁忌搜索算法优化评价指数公式中的参数,在提高搜索效率的同时避免陷人局部最优, 从而实现向全局最优解的快速收玫. 而禁忌列表长度的设置和模拟退火中退火形式的设置是影响算法收 玫速度的重要参数.

幂函数加和型富营养化综合指数是将多个具有可比性的单指标评价指数进行加和后取幂函数方式得 到的,因此,综合指数与分指数之间是线性和非线性关系,有较明确的物理意义.

优化好的湖泊富营养化综合评价公式适用于多项指标的评价,形式简单,易于计算,方便实用,能客观 地反映湖泊水质状况.

湖泊富营养化的综合评价旨在分析湖泊富营养化影响程度,提出改善水质环境及周边生态环境的有效 管理方式和措施，从而为开展环境污染和综合治理、环境规划及管理提供科学依据.

\section{6 参考文献}

[1] 古滨河. 美国湖 Apopka 湖的富营养化及其生态恢复. 湖泊科学, 2005, 17 (1) : $1-8$.

[2] Bernard P, Antoine L, Bernard L. Principal component analysis: an appropriate tool for water quality evaluation and management-application to a tropical lake system. Ecological Modeling, 2004, 178:295 - 311.

[3] 李祚泳, 丁 晶, 彭荔红. 环境质量评价原理与方法. 北京: 化学工业出版社, 2004: 87-93, 214$218,280-289$.

[4] 李祚泳, 张辉军. 我国若干湖泊水库的营养状态指数 TSIc 及其与各参数的关系. 环境科学学报, $1993,13(4)$ : $391-397$.

[5] 李祚泳, 彭荔红, 吕玉嫦. 基于遗传算法优化的普适卡森指数公式. 中国环境科学, 2001, 21 (2): $148-151$.

[6] 谢 平, 李 德, 陈广才等. 基于贝叶斯公式的湖泊富营养化随机评价方法及其验证. 长江流域资 源与环境, $2005, \mathbf{1 4}(2): 224-228$.

[7] 蔡庆华. 湖泊富营养化综合评价方法. 湖泊科学, 1997, 9(1)：89-94.

[8] Asic MD, Kovacevic-Vujcic VV. Taboo search methodology in global optimization. Computers and Mathematics with Application, 1999, 3(3): 125 - 133 .

[9] Belarmino A denso-Diaz. Restricted neighborhood in the taboo search for the flow shop problem. European $J$ of Operation Research. 1992, 62: 27 - 37.

[10] 刘宝坤, 王冬梅, 童 刚. Windows 环境下的禁忌搜索法解 Job-shop 问题. 自动化与仪表, 2000, 15 (1) : $65-66,71$.

[11] 曹立斌,周建兰. 一种改进的禁忌搜索法在函数优化问题中的应用. 微机发展, $2003,13: 39-42$.

[12] 徐 宁, 周尚波, 张红民等. 一种混合混沌优化方法及其应用. 系统工程与电子技术, 2003, 25(2): $226-227,244$.

[13] 倪长健, 丁 晶, 李祚泳. 免疫进化算法及其在暴雨强度公式参数优化中的应用. 长江科学院院报, $2002,19(6): 59-61$.

[14] 金相灿，屠清瑛. 湖泊富营养化调查规范 (第二版). 北京: 中国环境科学出版社, 1990: $286-302$.

[15] 甘义群, 郭永龙. 武汉东湖富营养化现状分析及治理对策. 长江流域资源与环境, 2004, 13(3): 277 -281 .

[16] 李凡修, 辛 焰, 陈 武. 集对分析用于湖泊富营养化评价研究. 重庆环境科学, 2000, 22(6): 10$11,16$. 